Bookreviews

\title{
FORTSCHRITTE DER BOTANIK
}

Springer-Verlag, Berlin, Heidelberg, New York 1971, Bd. 33, 387. S., 11 Abb.

Der bereits 33. Band der „Fortschritte der Botanik“ umfasst. die neueste botanische Literatur für dio Jahre 1969 und 1970 und gegebenenfalls auch ältere Arbeiten. Ebenso wie die vorhergehenden Ausgaben wird hier besonders eingehend über Forschungsergebnisse europäischer Wissenschaftler berichtet, obwohl auch bedeutende Artikel amerikanischer, australischer und asiatischer Zeitschriften Beachtung finden. Bei Abhandlungen, die sich mit allgemeinen botanjschen Problemen befassen, kann man mit wenigeren literarischen Hinweisen von aussereuropäischen Kontinenten auskommen. Bei den Kapiteln, die Fortachritte in der Systematik und Geobotanik beschreiben, muss man vorsussetzen, dass die angeführte Bibliographie nur einen Teil der tatsächlichen literarischen Produktion und der erzielten Ergebnisse erfasst. Die in keinen Weltsprachen veröffentlichte Literatur wird begreiflicherweise nur ausnahmswoise exzerpiert. Wissenschaftliche Ergebnisse tschechischer und slowakischer Botaniker werden an vielen Stellen dieses Buches kommentiert, was einerseits von einem guten Niveau dieser Arbeiten zeugt, andererseits auch deswegen, weil viele dieser Arbeiten in Weltsprachen veröffentlicht werden.

Der Teil ,Anatomie und Morphologie" (44 Seiten) befasst sich mit der Kern- und Zellteilung, mit der submikroskopischen Cytologie der Pilzzelle und der Morphologie und Anatomie des Blattes und der Blüte. Das letztgenannte Thema wurde von Prof. H. Weber und Prof. W. Troll ausserordentlich eingehend bearbeitet; es scheint, dags der Bereicherung dieser Fächer ein Studienergebnis einer viel breiteren Skala der untersuchten Taxa, insbesondere tropischer Sippen, zugrundeliegt.

Der Teil „Physiologie“ ist in diesem Band am umfangreichsten (123 Seiten) und enthält die Kapitol über die Elektrophysiologie der Zelle, den Wagserumsatz, die Stoffbewegungen, Minera]stoffwochsel, Stoffwechsel enorganiseher N-Verbindungen, Biosynthese der Polypektiden und Polyinen, Wachstum, Entwicklungsphysiologie und über die Bewegungen der Pfianzen. Das letztgenannte Thema befasst sich detailliert mit den neuen Ansichten über die Aufgabe von Statolithen; dio umfangreiche Diskussion zeugt davon, dass diese, vom verstorbenen Akademiker N.MEC entdeckten Organe auch weiter den Gegenstand eines lebhaften Interesses bilden.

Der Teil „Genetik" (60 Seiten) behandelt die Replikation, Rekombination, Funktion der Gene und die extrakaryotische Vererbung. In diesem die Genenfunktion beschreibenden Kapitel begegneten die deutschen und österreichischen Fachleute, die den grössten Teil dieses Kapitels im 33. Band der ,Fortgchritte der Botanik" belegen, einen gleichwertigen Mitarbeiter in Prof. F. K. ZimmermanN von der Universität in New York.

Der Teil ,Systematik“ (70 Seiten) enthält ein hervorragendes Referat über die Systematik und die Evolution der Semenpflanzen, in dem Prof. Ekrexidorfer und Dr. Fischer aus Wien in sehr gedrängter Form morphologische, phytochomische, cytologische, genetische und physiologische Beweise über verwandtschaftliche Beziehungen von Samenpfianzen zusammenfassen; dieseв Kapitel enthält 24 Seiten abgekürzter literarischer Hinweise! Im Teil ,Systematik" ist auch eine umfangreiche Utbersicht über die neuesten Forschungsergebnisse der Paleobotanik.

Der Abschnitt "Geobotenik“ (50 Seiten) wurde unter der Redaktion von Prof. EllenBerG in die klassischen Kapitel der floristischen, historischen, soziologischen und ökologisehen Geobotanik eingeteilt. Diese Kapitel hängen teilweise zusammen, was jedoch keine Duplizitäten hervorrief. Der floristische Teil enthält viele neue Angaben über neueste Florenwerke und Arealkarten. Der ökologische Teil behandelt den Einfluss der Wärme, des Lichtes, des Wassers und der Nährstoffe auf das Wachstum und die Verbreitung der Pflanzen.

Prof. F. Wetrstein, der Begründer dieser Reihe der ,Fortschritte der Botanik“ hat sich die heutige Flut von botanischen Zeitschriften und Informationen kaum vorstellen können. Es ist bewunderungswürdig, dass die heutigen Herausgeber und die Autoren des 33 . Bandes einen Modus fanden, wie sie die Leser über die Fortschritte in der Botanik so schnell und zeitgerecht informieren können.

JAN JENfK

Botanisches Institut der Tschechoslowakischen Akademie der Wissenschaften, 25243 Prủhonice bei Praha 